\title{
Four Simple Ward Based Initiatives to Reduce Unnecessary In-Hospital Patient Stay: A Quality Improvement Project
}

\author{
Asad Shabbir, Gorav Wali, Alan Steuer \\ Wexham Park Hospital
}

\begin{abstract}
Prolonged hospital stay not only increases financial stress on the National Health Service but also exposes patients to an unnecessarily high risk of adverse ward events. Each day accumulates approximately £225 in bed costs with additional risks of venousthromboembolism, hospital acquired infections, prescription errors, and falls. Despite being medically fit for discharge (MFFD), patients awaiting care packages with prolonged length of stay (LOS) have poorer outcomes and experience increased rates of mortality as a result.
\end{abstract}

A six cycle prospective audit was carried out to investigate if four simple ward based initiatives could optimise patient flow through a medical ward and reduce LoS of inpatients awaiting social packages and placement. The four daily initiatives were:

1. A morning board round between nurses and doctors to prioritise new or sick patients for early review.

2. A post ward round meeting between the multidisciplinary team to expedite rehabilitation and plan discharges early.

3. An evening board round to highlight which patients needed discharge paperwork for the next day to alleviate the wait for pharmacy.

4. A 'computer on wheels' on ward rounds so investigations could be ordered and reviewed at the bedside allowing more time to address patient concerns.

A control month in August 2013 and five intervention cycles were completed between September 2013 and January 2014. Prior to intervention, mean time taken for patients to be discharged with a package of care, once declared MFFD, was 25 days. With intervention this value dropped to 1 day. The total LoS fell from 46 days to 16 days. It was also found that the time taken from admission to MFFD status was reduced from 21 days to 15 days.

In conclusion this data shows that with four simple modifications to ward behaviour unnecessary inpatient stay can be significantly reduced.

\section{Problem}

The unselected general medical take has a high intake of elderly patients, a large proportion of whom were previously independent. The natural progression of their hospital stay is dictated by the general medical teams, and the multidisciplinary teams with which they work. A further smaller population of these patients go on to require either a residential home or nursing home placement. As the population ages it is hardly surprising that the burden on community placements is becoming stretched and this can be observed on the medical wards with many patients waiting significant lengths of time till community beds and packages of care become available. This problem is more significant in district general hospitals and with longer inpatient stays comes additional risks of adverse ward events.

\section{Background}

Prolonged inpatient stay accumulates approximately £225 per day in bed costs alone and the financial strain on the NHS is significantly stretched given the increasingly ageing population.[1] Despite further funding being signposted for care of the elderly specialities and their continuing care in the community, NHS trusts are increasingly struggling with a large volume of patients awaiting either residential or nursing home placement. The implications of this are threefold. Firstly the total number of bed-space occupied on general medical wards increases and forces trusts to either cancel elective admissions or divert at the emergency point of care. The second implication of this is that these patients spend large periods of time on the wards which increases their risk of adverse ward events and subsequent mortality.[2] These possible events range from prescription errors to falls, fractures, venousthromboembolic events and hospital acquired infections.[3] Finally it has already been established that longer inpatient stays are associated with an increased rate of re-admission and hospitalisation.[4] The aim of this project was to minimise ward delays as much as possible by using four simple ward behaviour modifications in an effort to speed up the safe discharge of elderly patients.

\section{Baseline measurement}


Prior to the planning of intervention strategies, a one month prospective data collection exercise was carried out in which we investigated the number of days taken for a patient to be made medically fit for discharge (MFFD), the time taken for a patient to be subsequently discharged to a placement and finally the total length of stay (LoS). This occurred in August 2013 between Monday and Friday. These three domains provided us with sufficient information to accurately assess the patients trajectory in hospital and with further audit cycle loops we continued to use these datum points as separate endpoints.

The time taken to be declared MFFD highlights the average time taken for the patient to be seen by a senior physician, for plans to be mobilised and treatments to be completed. The average times of length of stay were taken from a general medical ward with up to 28 patients. Those patients not requiring care packages were excluded from the study. This particular endpoint highlighted potential delays in the organisation and review of imaging and blood results. It was therefore decided that the first of our four ward based initiatives to help streamline safe discharge should be to use a 'computer on wheels.' The goal of this was to allow the individual completing the round to spend as much time as possible at the bedside whilst addressing patients concerns. At baseline the average time taken to be declared MFFD was 21 days (11.56-29.87, 95\%Cl).

The average number of days awaiting a discharge to a residential or nursing home having been made MFFD was 25 days (10.6-39.96, 95\%Cl). We observed several potential delays which prevented the earlier discharge to care centres. The junior doctor rota can have implications on the continuity of care on wards, especially in particularly busy firms and therefore a morning handover meeting was adopted to highlight potentially unwell patients for senior review. This also gave the opportunity for junior doctors to ensure that all team members were aware of outstanding jobs so they could be completed early in the day. A further meeting between multidisciplinary team members was introduced which would take place after the ward round and this would involve members of the social work team. During this one month observation period we found it challenging to discuss early placement without social workers and so this meeting was designed so that plans for safe discharge could be made early in the patient's stay to avoid delays in starting a care package. The total LoS, which measures the entire length of admission, was found to be 46 days $(25.9-66.1,95 \% \mathrm{Cl})$. In addition to the above mentioned strategies a final board round was introduced at the end of the day to ensure jobs and electronic discharge summaries were completed early to allow pharmacy sufficient time to organise medications.

See supplementary file: ds5365.docx - "Figure 1. Work plan during five month audit period"

\section{Design}

A strategy meeting was held between senior ward physicians, nursing staff, matrons, and IT members to ensure all were aware and in agreement with the proposed changes to the ward. Having identified several potential rate limiting steps in the safe discharge of elderly patients, the four ward based initiatives were introduced over a five month period from September 2013 to January 2014. A work plan was created which introduced the initiatives. Figure 1 below shows the work plan adopted daily during the five month audit period.

Figure 1. Work plan during five month audit period

Between September and January the above work plan was adhered to and a meeting was held between senior and junior doctors to ensure that all team members were aware of the audit process. The local IT department secured funding for the computer on wheels and supplied the ward with the hardware. Data was collected prospectively and analysed using the previously declared end points.

\section{Strategy}

Improvement (PDSA) cycle 1: Having identified the problems and objectives from the baseline observations in August 2013, the four ward based interventions were introduced from the very first audit cycle. Adherence from the outset was good and the 'computer on wheels' functioned appropriately. Data was collected from all medical ward rounds and feedback from the multidisciplinary team was excellent. Ward clerks and senior nursing staff were also encouraged to attend hand over meetings and help in the completion of ward jobs so that there was no delay in starting the midday and evening meetings.

Improvement (PDSA) cycle 2: New junior nursing staff and social work team members transferred into the ward, however the process was highlighted to them and they continued to attend all multidisciplinary meetings and they were reminded that they should attend all meetings. A roll-call at the beginning of each and every meeting was performed to ensure that all members were present. $A$ member of the IT department was also elected to join the hand over meetings to chair and ensure each patient was discussed during the three structured meetings throughout the day. This approach was adopted for the remaining cycles.

Improvement (PDSA) cycle 3: No further changes were implemented during the third cycle of data collection. Data continued to be collected appropriately and the senior and juniors were positively engaging with the programme.

Improvement (PDSA) cycle 4: Junior doctors rotated onto their next posting and so new juniors came to the ward and the project formed part of their induction. Initially they found the meetings challenging to attend given the tight time constraints, however a senior presence was always present at the meetings and through better delegation and organisation the juniors had no further issues with attendance.

Improvement (PDSA) cycle 5: No additional changes were implemented during the final month of data collection and all four initiatives continued to be used.

\section{Results}


Data was collected prospectively over five months. Adherence to attendance of the three scheduled meetings and the use of the 'computer on wheels' was excellent and the feedback from the junior and senior staff on the ward, including members of the multidisciplinary team, was excellent.

After five months of data collection, having introduced all four interventions, it was found that the average length of time taken for a patient to become MFFD fell from 21 days $(11.56-29.87,95 \% \mathrm{Cl})$ to 15 days $(10.47-18.86,95 \% \mathrm{Cl})$. The number of days taken for patients to be discharged having been made MFFD fell significantly from $25(10.60-39.96,95 \% \mathrm{Cl})$ to $1(-0.80-2.47,95 \% \mathrm{Cl})$. The final endpoint measured, total LoS, also significantly fell from 46 $(25.90-66.10,95 \% \mathrm{Cl})$ to $16(12.27-18.73,95 \% \mathrm{Cl})$. Below is a table which shows the variation of the measured endpoints, the raw data and box and whisker plots measuring each end point. (figure 2).

See supplementary file: ds5573.pdf - "Data set"

\section{Lessons and limitations}

The ward initiatives have had a positive impact on both the time taken for patients to become MFFD and their total LoS by reducing the time taken to organise impatient investigations and social placements. We have successfully shown an improvement in the flow of medical patients through the ward and have demonstrated that these changes can be maintained over a medium to long term basis.

The 'computer on wheels' allowed the medical team to not only order and review tests at the bedside, it improved the efficiency of the ward round by liberating more time to be spent addressing patients questions and concerns.

The pre and post ward round meetings ensured that all members of the multidisciplinary team were updated with the medical plans and successfully ensured that the most unwell patients were seen first so that their investigations and treatment plans could be prioritised. The post round social meeting also encouraged valuable input from community social workers so that community support and placement could be planned well in advance and once the patient was declared MFFD they could safely leave the ward to continue their rehabilitation in the community. The evening meeting was most useful when organising discharge paperwork and ensured that prescriptions were in pharmacy before the end of the day.

There were however several challenges faced by the medical team during the process. Initially the junior medical staff found it challenging to complete the required tasks before the next meeting, however with appropriate delegation of jobs, coordination between team members, and time management this did not remain an issue. All juniors kept within the European Working Time Directive (EWTD) guidelines and training was not negatively impacted on throughout the scheme.

In terms of future considerations for projects, the patients perspective was not objectified through formal feedback and although significant data already exists regarding the satisfaction of patients and length of stay, this could be explored.[5] In addition to this, some NHS trusts have 'seven day working' and although small single centre studies have demonstrated a positive correlation with numbers of Consultant ward rounds per week and inpatient stay and mortality, this could also be investigated with particular regard to district general hospitals.[6]

\section{Conclusion}

Several factors which slow the rate of ward discharges to community placement were identified and through the initiation of four simple initiatives the average time taken to discharge elderly patients with packages of care was significantly reduced. Effective teamwork, time management, and careful work plans with early social work input show that these changes are both achievable and sustainable.

\section{References}

1. NHS Institute for innovation and improvement, Length of Stay - Reducing Length of Stay, 2012, Accessed 15th May 2015,

[http://www.institute.nhs.uk/quality and service improveme nt_tools/quality_and_service_improvement_tools/length_of stay.html]

2. A Clarke, Why are we trying to reduce length of stay? Evaluation of the costs and benefits of reducing time in hospital must start from the objectives that govern change, Qual Health Care, 1996, 5(3):172-179.

3. A Clarke, Length of in-hospital stay and its relationship to quality of care, Quality and Safety in Health Care, 2002, 11(3):209-10.

4. Dr Foster's case notes, Length of hospital stay and subsequent emergency readmission, British Medical Journal, 2005, 331:7513(371).

5. Storm-Versloot $\mathrm{M}$, Vermeulen $\mathrm{H}$, van Lammeren $\mathrm{N}$ et al., Influence of the Manchester triage system on waiting time, treatment time, length of stay and patient satisfaction; a before and after study, Emergency Medicine Journal, 2014, 31:13-18.

6. Singh S, Lipscomb G, Padmakumar K et al., Daily consultant gastroenterologist ward rounds: reduced length of stay and improved inpatient mortality, Postgraduate Medical Journal, 2012, 88:583-587.

\section{Declaration of interests}

Nothing to declare

\section{Acknowledgements}

Nil

\section{Ethical approval}


BMJ Quality Improvement Reports

Ethics approval was obtained from the local ethics and audit committee at Wexham Park Hospital, Berkshire. 